\title{
Magnetic Properties of Co 2D Antidot Arrays with Hexagonal Holes Using Colloidal Crystal Template Method
}

\author{
Hong-Fei $\mathrm{Qi}^{1} \cdot$ Da-Bo Liu ${ }^{1} \cdot$ Song-Yan Dai ${ }^{1} \cdot$ Tian-Min Wang ${ }^{2}$
}

Received: 8 November 2015/Revised: 25 January 2016/Published online: 12 March 2016

(C) The Chinese Society for Metals and Springer-Verlag Berlin Heidelberg 2016

\begin{abstract}
In this work, Co 2D antidot arrays with well-ordered hexagonal holes ranging in size from 1.3 to $6.2 \mu \mathrm{m}$ were fabricated by colloidal crystal template method. The geometry configuration and magnetic properties of the samples were investigated. An enormous increase in coercivity and squareness, as compared with the corresponding continuous film, was observed. Magnetic properties of Co 2D antidot arrays showed a close dependence on pore diameter. According to geometric calculation, the effect of the pore diameter on magnetic properties was also discussed. The Co 2D antidot arrays obtained by this method are promising candidates for high-density information storage.
\end{abstract}

KEY WORDS: Co antidot arrays; Colloidal crystal template; Hexagonal holes; Magnetic properties

\section{Introduction}

Ordered magnetic structures, such as dots [1], wires [2], antidot arrays [3], and mesoporous colloidal crystals [4], are currently the focus of scientific interest due to their potential application for ultrahigh-density information storage and sensor devices. Antidot arrays are particularly of interest due to the absence of the superparamagnetic limit [5]. Typical methods developed in the past for fabricating magnetic antidot arrays are e-beam lithography [6], focused ion beam milling [7], X-ray lithography [8], ultraviolet lithography [9], porous membranes of anodic alumina templates [10], and colloid crystal templates [11]. Among them, the method of using colloid crystal templates

Available online at http://link.springer.com/journal/40195

Hong-Fei Qi

qhfei@sina.com

1 Department of Steel and Rare-Noble Metals, Beijing Institute of Aeronautical Materials, Beijing 100095, China

2 Center of Condensed Matter and Material Physics, Beihang University, Beijing 100191, China has several advantages such as simplicity, well-defined geometries, low cost, and large area fabrication feasibility [12].

Recently, magnetic antidot arrays with various microstructures and submicrostructures like rectangular hole [13], round hole [14], and elliptical hole [15] have been fabricated to explore novel phenomena and potential applications, which offer a possibility of stabilizing the magnetization distribution against the limits associated with the film magnetocrystalline anisotropy. These studies reveal that the geometries of the periodic holes can largely influence its magnetic behaviors since the holes introduce shape anisotropies allowing an enhanced coercivity compared with that of the continuous film.

The structures/shapes of the domain configuration are critical to the innovative functions of the porous materials [16]. Thus, the fabrication of desired architectures is an exciting area of research. Until now, few articles dealt with the magnetic properties of antidot array films with hexagonal holes, and none focused on the magnetic properties, such as coercivity and squareness, varying with pore diameter. In this study, we fabricate Co antidot array films with well-ordered hexagonal holes ranging in size from 1.3 to $6.2 \mu \mathrm{m}$ by polystyrene (PS) colloidal crystal template 
method. The dependence of magnetic properties on the geometric structure and pore size of the Co antidot arrays is also investigated.

\section{Experimental}

Three procedures were used to fabricate Co 2D antidot arrays onto $\mathrm{Si}(100)$ substrates $(1 \mathrm{~cm} \times 1 \mathrm{~cm} \times 0.1 \mathrm{~cm})$. Firstly, monodisperse polystyrene (PS) latex spheres with various diameters of 1.7, 3.0, 4.1, 5.8, 7.0, and 8.1 $\mu \mathrm{m}$ were synthesized by dropwise feeding procedure as described previously [17]. Secondly, the newly made spheres were first assembled into a close-packed monolayer on a vertical water surface, and then, the monolayer was transferred onto the Si substrates by a withdrawer at a certain lifting rates. More details of the template preparation method are described in Ref. [18]. Thirdly, antidot arrays were deposited by magnetron sputtering from a cobalt target at room temperature. The deposition was carried out under a high-purity $\mathrm{Ar}$ atmosphere with $1.5 \mathrm{~Pa}$ and the gas flow rate of $30 \mathrm{~mL} / \mathrm{min}$. Sputtering power and deposition rate were kept at $180 \mathrm{~W}$ and $1.2 \mathrm{~nm} / \mathrm{s}$, respectively. The sputtered samples were then supersonic vibrated and washed in a tetrahydrofuran solution to obtain the expected structure of Co films. Hexagonal macropores with various diameters of $1.3,2.2,3.1,4.3,5.2$, and $6.2 \mu \mathrm{m}$ were fabricated through repetition of the above procedure. In addition, Co continuous film was also deposited under the same condition for the convenience of comparison of magnetic properties.

The morphology of the sample was investigated by FEISIRION scanning electron microscopy (SEM) and CSPM4000 atomic force microscopy (AFM). X-ray diffraction (XRD) using $\mathrm{Cu} K_{\alpha}$ radiation was carried out to analyze the crystallographic structure of the Co film. And the magnetic properties were measured using Lake Shore7410 vibrating sample magnetometer (VSM) at room temperature.

\section{Results and Discussion}

\subsection{Microstructure and Morphology}

Figure 1 shows the typical SEM images of Co antidot arrays with pore diameters of 1.3 and $6.2 \mu \mathrm{m}$. Here, Fig. 1a shows the evidence the high hexagonal order in the antidot arrays, which mirrors the former positions of the voids between the spheres. As shown in Fig. 1b, each edge of hexagonal pore is nearly $3.1 \mu \mathrm{m}$, which is just half of the hole diameter $(\approx 6.2 \mu \mathrm{m})$. The antidot arrays exhibit regularly hexagonal macropores and good uniformity in both the size and the distance between the pores. A specially magnified cell of Co antidot arrays is shown in Fig. 1c, where mean hole diameter $(\approx 1.3 \mu \mathrm{m})$ and typical interhole distance $(\approx 360 \mathrm{~nm})$ are presented. This result indicates that the mean interhole distance to the diameters of corresponding PS particles is about $1 / 4$. Similar results have been observed in the other samples prepared with the same procedure, showing the generality of the geometric characteristics. In this article, when the sputtering power is suitable, the Co atoms can pitch in the interface of the adjoining microspheres along the tangential direction, which results in the hexagonal holes.

The hole depth of Co antidot arrays is measured by AFM imaging. As shown in Fig. 2, the mean hole depth of the $1.3 \mu \mathrm{m}$ and the sample is about $35 \mathrm{~nm}$, and the regular hexagonal holes are confirmed again. Furthermore, the depth values are nearly constant for different samples, indicating that all the samples are approximately the same thickness.

Figure 3 reveals the XRD spectrum of continuous Co film of the same thickness. Only two diffraction peaks correspond to the (111) and (110) of Co can be found in the pattern. The (111) peak is dominate, implying the obviously preferred orientation in Co grains of the antidot arrays. The average crystallite size $D$ of Co can be given by Scherrer's equation [19]:

$D=0.89 \lambda / \beta \cos \theta$,

where $\lambda$ is the X-ray wavelength and $\beta$ is the full width at half maximum (FWHM) of a diffraction peak at $2 \theta$ corrected for instrumental broadening. From Eq. (1), the estimated crystallite size of Co is about $17 \mathrm{~nm}$.

\subsection{Magnetic Properties}

The magnetic properties of the Co antidot arrays are measured at room temperature, and the typical results are indicated in Fig. 4. The $H_{\|}$represents the external magnetic field parallel to the surface of the Co antidot arrays, and the $H_{\perp}$ perpendicular to the surface of the arrays. It can be found that the value of the remanence of $H_{\|}$is $9.5 \mathrm{Am}^{2} /$ $\mathrm{kg}$, which is as much as 10 times greater than that of $H_{\perp}$. At the same time, the value of magnetization appears to be saturate at $306.7 \mathrm{~A} / \mathrm{m}$ for $H_{\|}$orientation. In contrast, the magnetization is difficult to saturate throughout the period in the case of $H_{\perp}$ orientation. Moreover, the same tendency also is found to the other samples. Therefore, it is confirmed that the easily magnetized direction is parallel to the surface of the samples and the Co antidot array has obvious magnetic anisotropy.

In order to investigate the effect of the pore diameter on the magnetic properties, the change of the coercivity and squareness at $H_{\|}$orientation as a function of the pore 

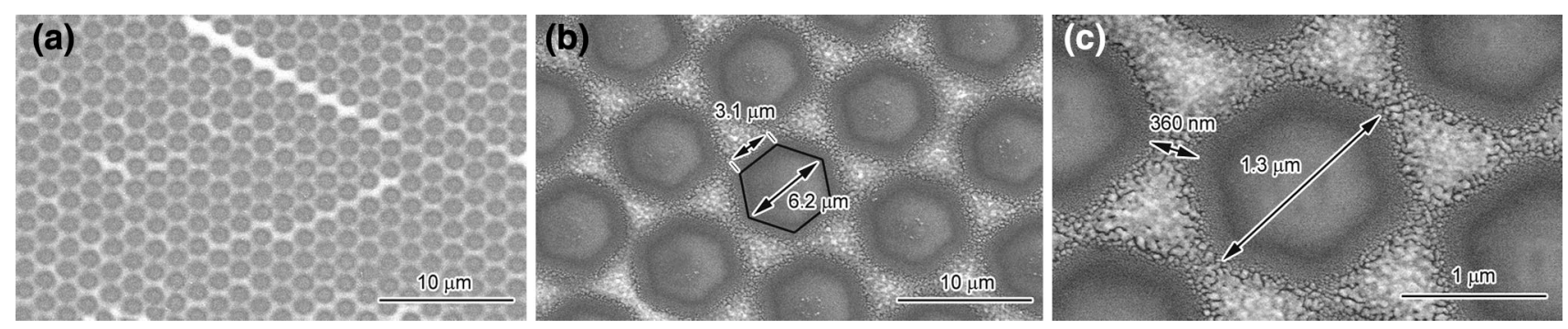

Fig. 1 Highly ordered Co antidot arrays with pore diameter about $1.3 \mu \mathrm{m} \mathbf{a}, 6.2 \mu \mathrm{m} \mathbf{b}$, c magnification of a displaying mean hole dimension and the angle-to-angle distances
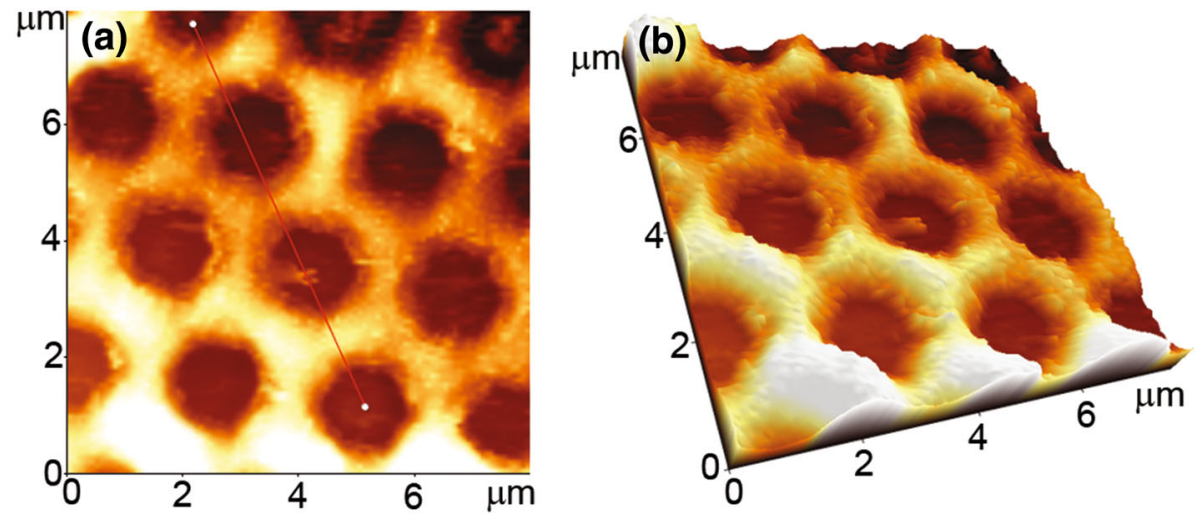

Fig. 2 a AFM image of Co antidot arrays with pore diameter about $1.3 \mu \mathrm{m}$, b the same image was converted into a three-dimensional perspective

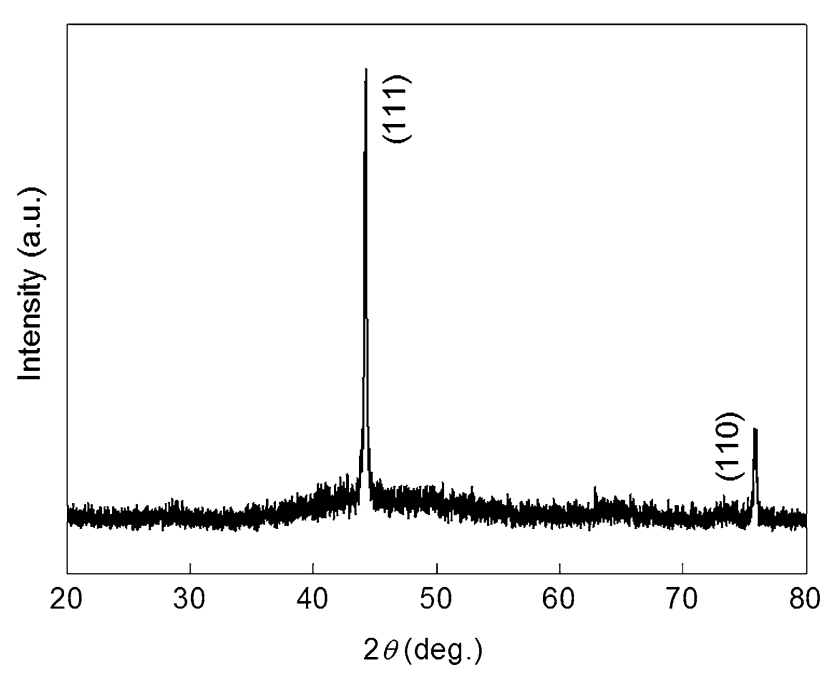

Fig. 3 XRD pattern of the continuous Co film

diameter is summarized in Fig. 5. Since the intervals of the pore diameters are close to micron scale, the dependence of the coercivity and squareness on the pore diameter is well observed. The coercivity decreases from 51.4 to $29.8 \mathrm{~A} / \mathrm{m}$, and the squareness decreases from 0.84 to 0.75 with increasing pore diameter from 1.3 to $6.2 \mu \mathrm{m}$. As indicated

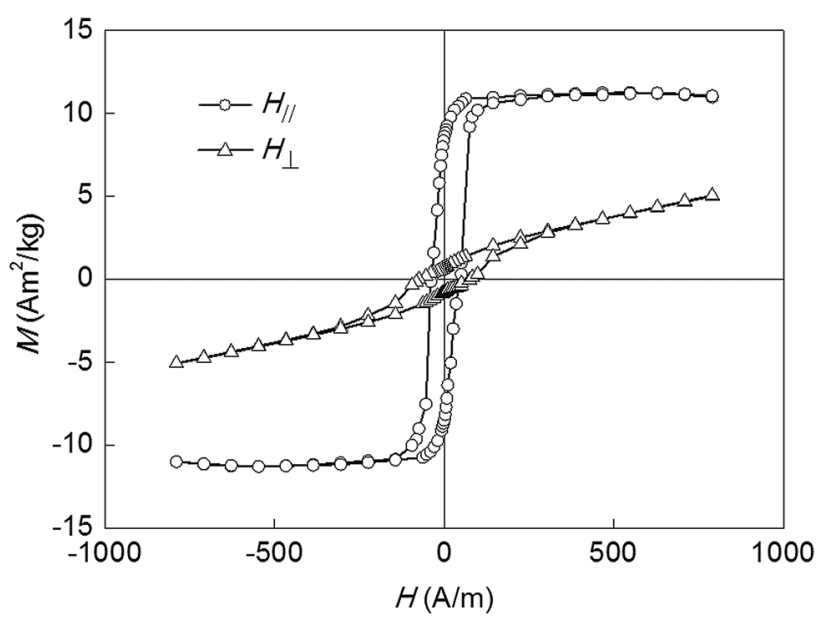

Fig. 4 Hysteresis hoops of the Co antidot arrays with pore diameter about $1.3 \mu \mathrm{m}$. The $H_{\|}$represents the direction of magnetic field applied parallel to the surface of Co antidot arrays and the $H_{\perp}$ perpendicular to Co antidot arrays

in Fig. 6, the values of coercivity and squareness of the Co films are the lowest, 0.9 and $0.31 \mathrm{~A} / \mathrm{m}$, respectively. It should be noted that when the pore size is in the range of $1.3-6.2 \mu \mathrm{m}$, the squareness values of the samples are close to the optimum value from media noise viewpoint [20]. 


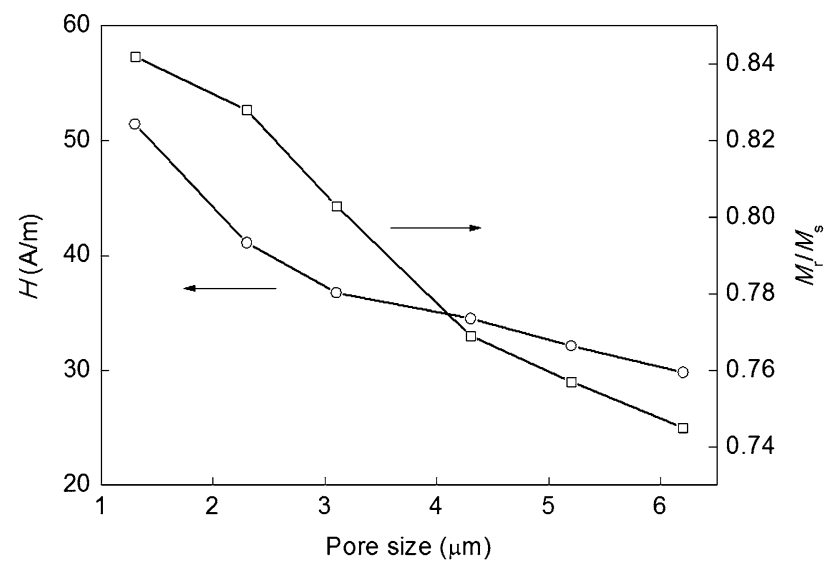

Fig. 5 Change of the coercivity $(H)$ and squareness $\left(M_{\mathrm{r}} / M_{\mathrm{s}}\right)$ at $H_{\|}$ orientation with the pore diameter

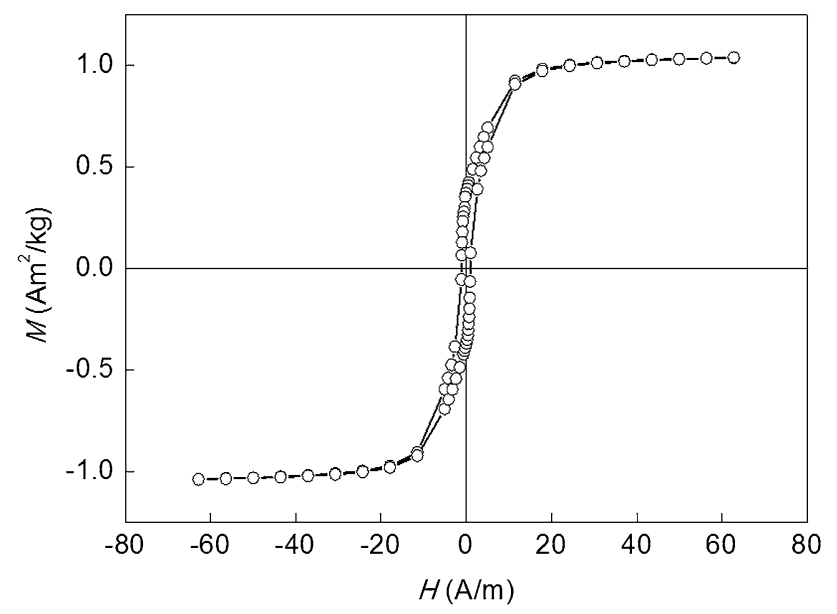

Fig. 6 Hysteresis hoops of the Co films at $H_{\|}$orientation

The difference of coercivity and squareness between continuous film and antidot arrays maybe comes from the shape anisotropies introduced by the hexagonal holes. For the case of Co film, the magnetic properties are mainly determined by the magnetocrystalline anisotropy, which makes the magnetic moment be along its easy-axis direction [21]. While in Co 2D antidot arrays, the patterninginduced shape anisotropies will make the magnetic moments be around the each edge of the hexagonal holes to decrease the demagnetization energy. This effect is similar to that the antidot pinned the magnetic moment $[22,23]$. Thus, we assume that the magnetic properties of Co 2D antidot arrays will be determined by the interplay between the intrinsic magnetocrystalline anisotropy and pinning of the hexagonal holes. In this case, only when the applied field is strong enough, the magnetic moment can be reversed to the direction of external field. Moreover, in the antidot arrays, the reversible component of magnetization may be much lower than that of continues films.
Accordingly, the values of coercivity and squareness of $\mathrm{Co}$ 2D antidot arrays are much higher than those of Co continues films.

Figure 7 illustrates a geometric model used to define the in-plane structural parameters of Co 2D antidot arrays with hexagonal holes. The fundamental building block of the antidot arrays is equilateral triangle, which side length is the diameter of the corresponding PS particles $(d)$. According to Fig. 1, the interhole distance $(a)$ and the radius of hexagonal macropore $(r)$ are $d / 4$ and $3 d / 8$, respectively. Therefore, the area of formed array surface surrounded by the triangle is $\left(37 \sqrt{3} d^{2}\right) / 256$ corresponding the area of the triangle surface $\sqrt{3} d^{2} / 4$, so the ratio of both areas is a constant and equals to 0.578 , no matter how much the pore diameter is. Therefore, the number of the hexagonal holes decreases while the amount of Co is thought to keep constant with the increase in the pore diameter. As a result, with the increase in the pore diameter, the pinning effect becomes increasingly weak due to the decrease in the shape anisotropies. This behavior leads to the situation of decreased number of the magnetic moments around the antidot and increased reversible component of magnetization. Therefore, both the coercivity and the squareness decrease with increasing pore diameter of Co 2D antidot arrays.

\section{Conclusions}

In summary, Co 2D antidot array films with well-ordered hexagonal holes ranging in size from 1.3 to $6.2 \mu \mathrm{m}$ were fabricated by PS colloidal crystal template method. The magnetic behaviors of both the antidot array films and the

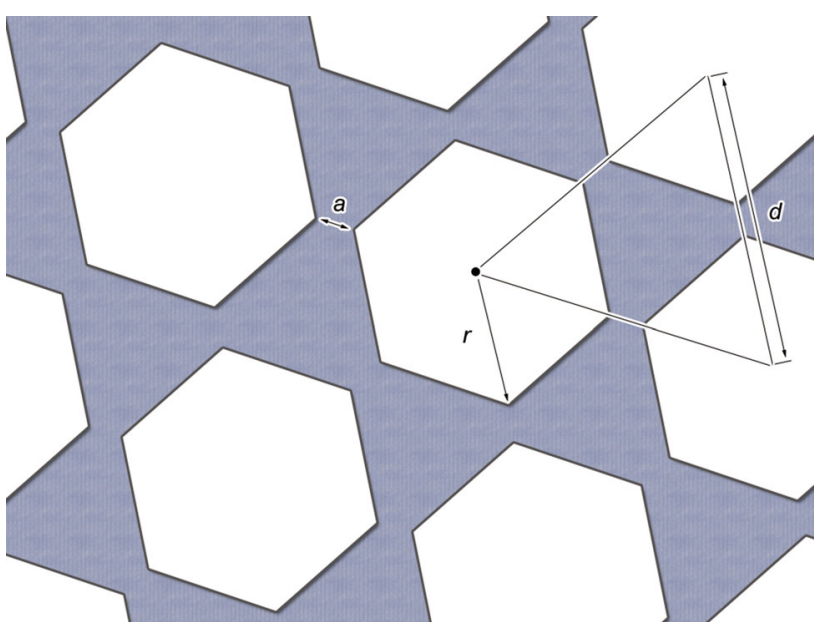

Fig. 7 Structure model of Co 2D antidot arrays with hexagonal holes, where $d$ is defined as the diameter of the corresponding PS particles, $r$ is the radius of the hexagonal macropore, and $a$ is the interhole distance 
continuous film were investigated at room temperature. All the structured films show a significantly enhanced coercivity and squareness in comparison with the unpatterned film, which could be attributed to the interplay between the intrinsic magnetocrystalline anisotropy and pinning of the hexagonal holes. In addition, with increasing the pore diameter, both the coercivity and the squareness of the Co 2D antidot arrays decrease gradually. According to geometric calculation, the shape anisotropies become increasingly weak with the increase in the pore diameter, which are thought to be responsible for the variation in the coercivity and the squareness. It is obvious that the Co $2 \mathrm{D}$ antidot arrays with well-ordered hexagonal holes are very promising for high-density information storage.

Acknowledgments This work was financially supported by the Innovation Foundation of Aviation Industry Corporation of China (No. JK65150307).

\section{References}

[1] V. Proshchenko, Y. Dahnovsky, Chem. Phys. 461, 58 (2015)

[2] Z. Chen, W. Wang, K.G. Zhu, Acta Metall. Sin. (Engl. Lett.) 28, 1 (2015)

[3] R.L. Rodríguez-Suárez, J.L. Palma, E.O. Burgos, S. Michea, J. Escrig, J.C. Denardin, C. Aliaga, J. Magn. Magn. Mater. 350, 88 (2014)

[4] J.E. Chen, H.Y. Lian, S. Dutta, S.M. Alshehri, Y. Yamauchi, M.T. Nguyen, T. Yonezawa, K.C.W. Wu, Phys. Chem. Chem. Phys. 17, 27653 (2015)

[5] S. Bedanta, W. Kleemann, J. Phys. D 43, 013001 (2009)
[6] L.Z. Liu, S.B. Tian, Y.Z. Long, W.X. Li, H.F. Yang, J.J. Li, C.Z. Gu, Vacuum 105, 21 (2014)

[7] M. Madami, G. Gubbiotti, S. Tacchi, G. Carlotti, S. Jain, Phys. B 435, 152 (2014)

[8] N.G. Deshpande, J.S. Hwang, M.S. Seo, Y.J. Yoo, J.Y. Rhee, K.W. Kim, Y.C. Shao, K.H. Chen, W.F. Pong, Y.P. Lee, Appl. Surf. Sci. 314, 453 (2014)

[9] S. Mallick, S. Bedanta, J. Magn. Magn. Mater. 382, 158 (2015)

[10] J.L. Palma, C. Gallardo, L. Spinu, J.M. Vargas, L.S. Dorneles, J.C. Denardin, J. Escrig, J. Magn. Magn. Mater. 344, 8 (2013)

[11] M. Coïsson, A. Manzin, G. Barrera, F. Celegato, E. Enrico, P. Tiberto, F. Vinai, Appl. Surf. Sci. 316, 380 (2014)

[12] J. Sklenar, V.S. Bhat, L.E. DeLong, O. Heinonen, J.B. Ketterson, Appl. Phys. Lett. 102, 152412 (2013)

[13] Y. Tsuchiya, H.T. Huy, T. Ishida, S. Pyon, T. Tamegai, Phys. C 504, 39 (2014)

[14] T.J. Meng, J.B. Laloë, S.N. Holmes, A. Husmann, G.A.C. Jones, J. Appl. Phys. 106, 033901 (2009)

[15] R. Zivieri, L. Giovannini, Photon. Nanostruct. 11, 191 (2013)

[16] V. Malgras, Q. Ji, Y. Kamachi, T. Mori, F.K. Shieh, K.C.W. Wu, K. Ariga, Y. Yamauchi, Bull. Chem. Soc. Jpn 88, 1171 (2015)

[17] H.F. Qi, W.C. Hao, H.Z. Xu, J.Y. Zhang, T.M. Wang, Colloid Polym. Sci. 287, 243 (2009)

[18] H.F. Qi, D.B. Liu, L.J. Teng, T.M. Wang, F. Luo, Y. Tian, Acta Metall. Sin. 50, 1163 (2014). (in Chinese)

[19] M. Yu, H. Qiu, X. Chen, Thin Solid Films 518, 7174 (2010)

[20] Y.L. Li, T.F. Feng, Z.Y. Chen, Appl. Surf. Sci. 257, 3666 (2011)

[21] H. Suzuki, K. Nakamura, T. Akiyama, T. Ito, Appl. Surf. Sci. 254, 7843 (2008)

[22] C.I.L. de Araujo, R.C. Silva, I.R.B. Ribeiro, F.S. Nascimento, J.F. Felix, S.O. Ferreira, L.A.S. Mól, W.A. Moura-Melo, A.R. Pereira, Appl. Phys. Lett. 104, 092402 (2014)

[23] X.K. Hu, S. Sievers, A. Müller, H.W. Schumacher, J. Appl. Phys. 113, 103907 (2013) 